ARTIGO ORIGINAL ORIGINAL ARTICLE

\title{
Avaliação de custo-efetividade do cateterismo em veia jugular interna guiado por ultrassom no Sistema Único de Saúde brasileiro
}

\author{
Evaluation of cost-effectiveness of internal \\ jugular vein catheterization by ultrasound in \\ the Brazilian National Health System
}

Carlos Eduardo Pessanha Boller ${ }^{1}$, Kátia Marie Simões Senna², Roberta da Silva Teixeira², Marcelo Correa Goulart², Bernardo Rangel Tura², Marisa da Silva Santos²

DOI: 10.21115/JBES.v11.n2.p112-8

\section{Palavras-chave: \\ cateterismo venoso central, ultrassonografia de intervenção, avaliação de custo- -efetividade, veias jugulares}

\section{Keywords:}

central venous catheterization, interventional ultrasonography, cost-effectiveness evaluation, jugular veins

\section{RESUMO}

Objetivo: Determinar a factibilidade econômica da técnica de cateterismo central em veia jugular interna guiada pelo ultrassom comparado à técnica-padrão, sob a perspectiva pagadora do Sistema Único de Saúde brasileiro. Métodos: Análise de custo-efetividade utilizando modelo de árvore de decisão sob uma população de pacientes adultos em um cenário de uma unidade terciária. Os custos diretos dos materiais e procedimentos foram estimados utilizando bancos de registros de compras nacionais. Os desfechos foram a ocorrência ou não da punção arterial acidental grave (principal complicação associada ao sítio de punção). Também foram conduzidas análises de sensibilidade determinística e probabilística, bem como curva de aceitabilidade. Resultados: A intervenção onerou o modelo em $\mathrm{R} \$ 53,81$. A razão de custo-efetividade incremental calculada foi de $\mathrm{R} \$ 17.936,66$ por complicação grave evitada e a curva de aceitabilidade evidenciou que a técnica é custo-efetiva sob uma intenção de pagar de R\$ 18.125,00. Na análise de sensibilidade probabilística, 63,6\% das simulações mostraram-se custo-efetivas. Conclusão: A intervenção é custo-efetiva, contribuindo para a redução das complicações graves, e o resultado pode proporcionar segurança para tomadas de decisões quanto à padronização do uso da ultrassonografia como orientador do procedimento.

\begin{abstract}
Objective: To determine the economic feasibility of the central catheterization technique in the internal jugular vein guided by the ultrasound compared to the standard technique, under the perspective of the Brazilian Unified Health System. Methods: Cost-effectiveness analysis using decision tree model under a population of adult patients in a tertiary unit scenario. The direct costs of the materials and procedures were estimated using banks of national procurement records. The outcomes were the occurrence or not of severe accidental arterial puncture (the main complication associated with the puncture site). Analyzes of deterministic and probabilistic sensitivity were also conducted, as well as acceptability curve. Results: The intervention cost the model in R\$ 53.81. The calculated incremental cost-effectiveness ratio was $\mathrm{R} \$ 17,936.66$ due to a serious complication avoided and the acceptability curve showed that the technique is cost-effective under an intention to pay $\mathrm{R} \$ 18,125.00$. In the probabilistic sensitivity analysis, $63.6 \%$ of the simulations were cost-effective. Conclusion: The intervention is cost-effective, contributing to the reduction of severe complications and the result can provide security for decision making regarding the standardization of the use of ultrasonography as a guideline of the procedure.
\end{abstract}

\footnotetext{
Recebido em: 11/06/2019. Aprovado para publicação em: 28/07/2019.

1. Fundação Oswaldo Cruz/Instituto Nacional de Saúde da Mulher, da Criança e do Adolescente Fernandes Figueira, Rio de Janeiro, RJ, Brasil.

2. Instituto Nacional de Cardiologia, Rio de Janeiro, RJ, Brasil.

Instituição onde trabalho foi executado: Instituto Nacional de Cardiologia, Rio de Janeiro, RJ, Brasil.

Informações sobre auxílios recebidos sob a forma de financiamento, equipamentos ou medicamentos: Pesquisa

realizada sem qualquer auxílio financeiro, de equipamento ou de medicamentos.

Congressos: A pesquisa em questão não foi apresentada em nenhum congresso até o momento.

Autor correspondente: Carlos Eduardo Pessanha Boller. Rua Assis Bueno, 12/605, Botafogo, Rio de Janeiro, RJ, Brasil.

CEP: 22280-080. Telefone: (21) 98794-0200.E-mail: kadu.boller@gmail.com
} 


\section{Introdução}

O cateterismo venoso central, caracterizado pela inserção de um dispositivo apropriado na obtenção do acesso vascular, acarreta problemas em aproximadamente $15 \%$ das tentativas (Merrer et al., 2001). Ressalta-se que, ao se evidenciar a necessidade de proceder a uma segunda tentativa, o risco de suceder danos é potencializado em 5,5 vezes (Mansfield et al., 1994). Entre essas complicações factíveis, estão as infecciosas, trombóticas e mecânicas, sendo a punção arterial acidental relatada como a de maior frequência (Kornbau et al., 2015), podendo ocorrer em 10\% dos procedimentos quando realizados na veia jugular interna (Brass et al., 2015).

Com o intuito de minimizar os eventuais problemas, instituições que normatizam aspectos da segurança do paciente, como a Agency for Health Care Research and Quality (AHRQ, 2001) e a National Institute for Clinical Excellence (NICE, 2002), recomendam o uso da ultrassonografia durante os procedimentos de cateterismo venoso central. Tal orientação é reiterada pelo suporte que a tecnologia propicia no adequado posicionamento do cateter no leito vascular, o que pode viabilizar a diminuição da quantidade de tentativas, bem como as falhas na realização do procedimento (AHRQ, 2001; NICE, 2002; Petisco et al., 2013).

Apesar dessa perspectiva promissora, o cateterismo guiado por ultrassom ainda não é universalmente adotado. Isso sucede pela não disponibilidade de equipamentos e por limitações financeiras. Em um cenário marcado por recursos escassos, é primordial ponderar o custo de agregar a tecnologia ao procedimento mediante a sua capacidade de reduzir complicações e falhas. Isso posto, a condução de um estudo de custo-efetividade pode colaborar na tomada de decisão dos gestores sobre a incorporação da referida técnica. No mais, é feita menção à escassez de avaliações econômicas sobre o uso da ultrassonografia como referência para a realização de cateterismo venoso central no cenário brasileiro. O presente estudo teve como objetivo determinar a factibilidade econômica da técnica de cateterismo central em veia jugular interna guiado pelo ultrassom comparado à técnica-padrão, sob a perspectiva pagadora do Sistema Único de Saúde (SUS) brasileiro, ou seja, em um cenário usualmente encontrado nas unidades públicas de saúde.

\section{Métodos}

Foi realizado um estudo de custo-efetividade, no qual a população-alvo foram pacientes adultos submetidos ao procedimento de cateterismo venoso central de veia jugular interna em unidades assistenciais de saúde (dentro e fora de unidades de terapia intensiva). O contexto abordado consiste na tomada de decisão sobre qual técnica deverá ser prioritária no procedimento de punção venosa profunda no âmbito analisado. A perspectiva adotada foi a do SUS como pagador único, por conseguinte bancos de dados públicos foram utilizados para a estimação dos custos analisados. As intervenções sob comparação foram a punção guiada por ultrassom e a orientada somente pelos marcos anatômicos, e essas escolhas foram fundamentadas tanto pelo fato de a ultrassonografia se apresentar como promissora quanto pelo fato de os marcos anatômicos serem utilizados usualmente como técnica-padrão na condução do cateterismo. Assim, a hipótese do estudo foi: "Em pacientes adultos submetidos à punção da veia jugular interna em uma unidade pública assistencial de saúde, a técnica de ultrassom guiada é custo-efetiva quando comparada à técnica-padrão".

Como horizonte temporal, o período de três dias após a realização do procedimento foi estabelecido, haja vista que, segundo o painel de especialistas, é o tempo necessário para diagnóstico e tratamento das potenciais complicações. Em virtude desse curto horizonte temporal, não foi adotada nenhuma taxa de desconto na avaliação econômica. Por fim, optou-se por selecionar como desfechos a ocorrência ou não da punção arterial acidental grave, dada a sua relevância evidenciada pelas informações disponíveis na literatura.

\section{Mensuração da efetividade}

Para verificar o conhecimento já existente sobre o uso da intervenção proposta, foi elaborada inicialmente uma busca estruturada na literatura de modo a identificar estudos que avaliassem o uso da ultrassonografia auxiliando o cateterismo venoso central de veia jugular interna e que pudessem fornecer dados vitais na elaboração e provimento do modelo utilizado no estudo. A busca na literatura, conforme descrito a seguir, foi realizada na base de dados eletrônica MEDLINE (Medical Literature Analysis and Retrieval System Online), por meio da interface de acesso PubMed em março de 2018.

A estratégia de busca ("Jugular Veins"[Mesh] OR jugular*[tiab]) AND ("Ultrasonography"[Mesh] OR ultrasonogra* OR doppler*[tiab] OR ultrasound*[tiab]) AND ("Central VenousCatheters"[Mesh] OR "Catheterization, Central Venous" [Mesh] OR catheter*[tw] OR acess*[tiab] OR central-line*[tw] OR cannulat*[tiab]) AND (systematic[sb]) foi elaborada com a inclusão de filtro para revisões sistemáticas e inicialmente sem restrição para idiomas. Após a seleção inicial, os critérios pertinentes aos estudos em idiomas diferentes do inglês, espanhol e português, e publicados há mais de 15 anos, foram tidos como de exclusão. Tanto essa seleção como a extração de dados foram realizadas por dois avaliadores independentes com discordâncias resolvidas por consenso.

Os desfechos identificados foram agrupados em procedimentos com base em análise da literatura e descritos como "sem complicação grave" e "punção arterial acidental grave". Especificamente para a esse último desfecho, os valores estatísticos obtidos na literatura para "punção arterial acidental" foram sumarizados em uma metanálise (Figura 1) utilizando o pacote Meta do software R, versão 3.1.2, obtendo-se uma frequência de 0,100. 


\section{Mensuração dos recursos e custos}

Os custos diretos do procedimento foram estimados atribuindo valores extraídos de bancos de dados nacionais, como o "Sistema de Gerenciamento da Tabela de Procedimentos, Medicamentos, Órteses, Próteses e Materiais" (Sigtap) e o Portal de Compras Governamentais do Governo Federal. Para o treinamento da intervenção no procedimento, o valor foi estabelecido mediante a comunicação pessoal a empresas que prestam esse tipo de serviço.

A moeda brasileira corrente (real) foi empregada e para definição dos custos do modelo, utilizando-se os seguintes períodos: (i) maio de 2018 para os custos extraídos do Sigtap; (ii) segundo bimestre de 2018 para os custos extraídos do portal de compras governamentais - devido a diversidade de valores e quantidades licitadas, foi considerada a mediana dos preços praticados nesse período; (iii) agosto de 2018 para o treinamento da intervenção no procedimento. Não foi necessário qualquer tipo de conversão na moeda, pois todos os valores estimados já estavam na moeda de referência deste estudo.

\section{Modelo de custo-efetividade}

A modelagem adotada fundamentou-se na árvore de decisão (Figura 2), em função dos desfechos serem de curto prazo não necessitando da realização de ciclos. Subsequente a todo o processo de identificação dos desfechos clínicos obtidos na literatura, eles foram validados por um painel de especialistas que assinalaram o prognóstico e a conduta para cada um dos casos. Logo, uma árvore de decisão foi elaborada no software TreeAge Pro, tendo como cenário fictício uma unidade de atendimento terciário de saúde cujo aparelho de ultrassom havia sido adquirido anteriormente e com equipe não possuidora de treinamento para utilizar o equipamento com a finalidade proposta.

Os pressupostos que serviram de base para o referido modelo foram: (i) "punção arterial acidental grave", que foi a única complicação grave considerada e requereu como conduta terapêutica sutura arterial cirúrgica e, consequentemente, duas diárias na unidade de terapia intensiva; (ii) "sem complicação grave", sendo agrupadas punções arteriais acidentais sem gravidade, demais complicações possíveis sem

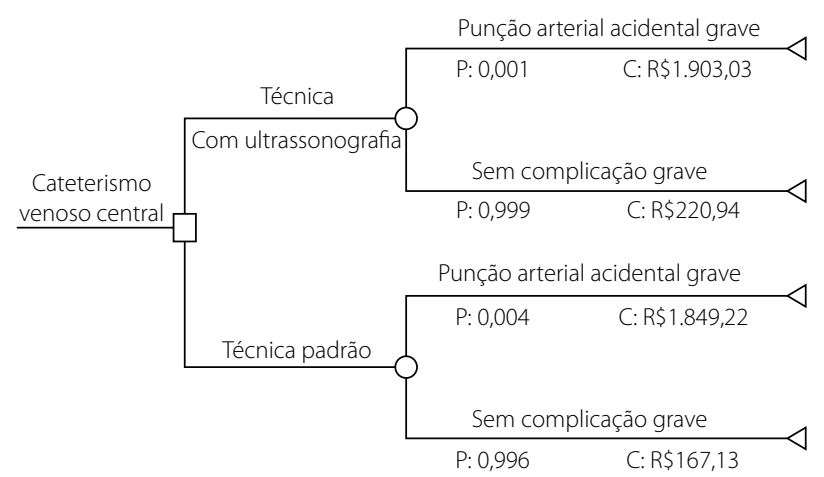

Figura 2. Árvore de decisão da técnica de cateterismo venoso central.



Figura 1. Incidência de punção arterial acidental quando realizada sob técnica de procedimento padrão 
relevância estatística individualmente e ausência de complicações, requerendo como conduta terapêutica para as duas primeiras dois curativos compressivos e para a última nenhuma conduta assistencial; (iii) "ausência de complicações", sendo demandada a utilização de apenas uma tentativa de inserção do cateter; (iv) para todas as demais complicações, foi considerada a necessidade de duas tentativas de cateterismo e, consequentemente, o uso de dois cateteres.

Destaca-se que os custos de compra e manutenção do equipamento foram desconsiderados, dado que a instituição, como descrito no cenário, possui previamente o equipamento para outros fins e, consequentemente, a unidade não será onerada por esses motivos. Para o manuseio dessa tecnologia, foi considerado um único operador, sendo esse o responsável pela realização do cateterismo.

No que concerne aos métodos analíticos, a análise do modelo também utilizou o software TreeAge Pro, tendo como parâmetros para análise de sensibilidade determinística uma variação de $50 \%$ do valor da média como limites superior e inferior, e para análise probabilística, o mesmo intervalo de valores, realizando 1.000 simulações de Monte Carlo. Essa variação em questão foi escolhida por proporcionar uma faixa mais abrangente e, assim, obter o real impacto das variáveis. No restante, menciona-se que na análise probabilística os valores das efetividades foram simulados utilizando uma distribuição beta e os custos, uma distribuição gama.

\section{Resultados}

Tomando por base os valores contidos nas bases de preço anteriormente apontadas e as composições detalhadas na Tabela 1, obtiveram-se como custos da punção arterial acidental grave e das complicações não graves, quando produzidas sob técnica-padrão, respectivamente, $R \$ 1.849,22$ e R\$ 167,13. Quando realizada com a técnica sob orientação da ultrassonografia, ambos os desfechos oneraram em $\mathrm{R} \$ 53,81$.

Ao se considerar o cálculo da probabilidade de uma "punção arterial acidental grave" (PAAG) na técnica-padrão como o produto entre as probabilidades de ocorrer uma punção arterial acidental $(0,10)$ e de ser grave $(0,04)$, obteve-se, fundamentado na literatura (Kusminsky, 2007), o valor de 0,004. Consequentemente, para a ocorrência de "sem complicação grave", o complemento é de 0,996. Na mensuração da probabilidade de ocorrência de PAAG, quando realizado sob a intervenção estudada, obteve-se pelo produto entre o comparador $(0,004)$ e o risco relativo entre as técnicas $(0,21)$ (Brass et al., 2015) o porte de 0,001 e, para "sem complicação grave", a estimativa, por meio do complemento, é de 0,999 (Tabela 2).

O custo e a efetividade incremental do procedimento utilizando a ultrassonografia foram mensurados em, respectivamente, R\$ 53,81 e 0,3\% (0,003). Portanto, para cada complicação grave evitada ao se realizar o procedimento com a intervenção proposta, a razão de custo-efetividade incremental (RCEI) foi de R\$ 17.936,66.
Tabela 1. Detalhamento dos dados de custos e probabilidades (input)

\begin{tabular}{|c|c|c|c|}
\hline \multicolumn{4}{|c|}{ Custos } \\
\hline Complicação & Valor & $\begin{array}{c}\text { Técnica com } \\
\text { ultrassono- } \\
\text { grafia (n) }\end{array}$ & $\begin{array}{c}\text { Técnica } \\
\text { padrão (n) }\end{array}$ \\
\hline
\end{tabular}

\begin{tabular}{|c|c|c|c|}
\hline \multicolumn{4}{|c|}{ Material/Procedimento (fonte) } \\
\hline \multicolumn{4}{|c|}{ Punção arterial acidental grave } \\
\hline $\begin{array}{l}\text { Cateterismo venoso } \\
\text { central }\end{array}$ & $R \$ 112,48$ & 2 & 2 \\
\hline $\begin{array}{l}\text { Diária de terapia } \\
\text { intensiva }\end{array}$ & $R \$ 508,63$ & 2 & 2 \\
\hline Ultrassonografia & $\mathrm{R} \$ 39,60$ & 1 & NA \\
\hline Capa protetora & $\mathrm{R} \$ 12,87$ & 1 & NA \\
\hline Sutura arterial cirúrgica & $R \$ 607,00$ & 1 & 1 \\
\hline $\begin{array}{l}\text { Treinamento do uso da } \\
\text { ultrassonografia }\end{array}$ & $\mathrm{R} \$ 1,34$ & 1 & NA \\
\hline
\end{tabular}

\begin{tabular}{llll}
\hline \multicolumn{4}{l}{ Sem complicação grave } \\
\hline \multicolumn{4}{l}{ Punção arterial acidental sem gravidade } \\
\hline $\begin{array}{l}\text { Cateterismo venoso } \\
\text { central }\end{array}$ & $\mathrm{R} \$ 112,48$ & 2 & 2 \\
\hline Curativo transparente & $\mathrm{R} \$ 1,90$ & 2 & 2 \\
\hline Luva estéril & $\mathrm{R} \$ 1,13$ & 2 & 2 \\
\hline Gaze & $\mathrm{R} \$ 0,39$ & 1 & $\mathrm{NA}$ \\
\hline Ultrassonografia & $\mathrm{R} \$ 39,60$ & 1 & $\mathrm{NA}$ \\
\hline Capa protetora & $\mathrm{R} \$ 12,87$ & 1 & $\mathrm{NA}$ \\
\hline $\begin{array}{l}\text { Treinamento do uso da } \\
\text { ultrassonografia }\end{array}$ & $\mathrm{R} \$ 1,34$ & 1 & $\mathrm{NA}$ \\
\hline
\end{tabular}

\begin{tabular}{llll}
\hline Demais complicações & & & \\
\hline $\begin{array}{l}\text { Cateterismo venoso } \\
\text { central }\end{array}$ & $\mathrm{R} \$ 112,48$ & 2 & 2 \\
\hline Ultrassonografia & $\mathrm{R} \$ 39,60$ & 1 & $\mathrm{NA}$ \\
\hline Capa protetora & $\mathrm{R} \$ 12,87$ & 1 & $\mathrm{NA}$ \\
\hline $\begin{array}{l}\text { Treinamento do uso da } \\
\text { ultrassonografia }\end{array}$ & $\mathrm{R} \$ 1,34$ & 1 & $\mathrm{NA}$ \\
\hline
\end{tabular}

\begin{tabular}{llll}
\hline \multicolumn{4}{l}{ Ausência de complicações } \\
\hline $\begin{array}{l}\text { Cateterismo venoso } \\
\text { central }\end{array}$ & $\mathrm{R} \$ 112,48$ & 1 & 1 \\
\hline Ultrassonografia & $\mathrm{R} \$ 39,60$ & 1 & $\mathrm{NA}$ \\
\hline Capa protetora & $\mathrm{R} \$ 12,87$ & 1 & $\mathrm{NA}$ \\
\hline $\begin{array}{l}\text { Treinamento do uso da } \\
\text { ultrassonografia }\end{array}$ & $\mathrm{R} \$ 1,34$ & 1 & $\mathrm{NA}$ \\
\hline
\end{tabular}

\begin{tabular}{lcc}
\hline \multicolumn{3}{c}{ Probabilidades } \\
\hline Probabilidades & Valor & Referência \\
\hline Punção arterial acidental & 0,10 & (metanálise) \\
\hline Gravidade & 0,04 & (Kusminsky, 2007) \\
\hline $\begin{array}{l}\text { Risco Relativo entre as } \\
\text { técnicas }\end{array}$ & 0,21 & (Petisco et al., 2013) \\
\hline
\end{tabular}

Legenda: $\mathrm{n}$ - quantidade; NA - não aplicável. 
Tabela 2. Detalhamento de dados de custos e probabilidades (output)

\begin{tabular}{lc}
\hline Variável & Valor \\
\hline Custos & \\
\hline Punção arterial acidental grave com ultrassonografia & $R \$ 1.903,03$ \\
\hline Punção arterial acidental grave com técnica padrão & $R \$ 1.849,22$ \\
\hline Complicações não graves com ultrassonografia & $R \$ 220,94$ \\
\hline Complicações não graves com técnica-padrão & $R \$ 167,13$ \\
\hline Custo incremental & $R \$ 53,81$ \\
\hline Probabilidades & \\
\hline Punção arterial acidental grave com ultrassonografia & 0,004 \\
\hline Punção arterial acidental grave com técnica-padrão & 0,001 \\
\hline Complicações não graves com ultrassonografia & 0,996 \\
\hline Complicações não graves com técnica-padrão & 0,999 \\
\hline Efetividade incremental & 0,003 \\
\hline
\end{tabular}

O resultado da análise de sensibilidade determinística, como observado pela Figura 3A, possibilitou a identificação das variáveis que impactaram na RCEl, sendo as principais "Probabilidade de punção arterial acidental grave, quando utilizada a técnica-padrão" (RCEl: R\$ 8.000,00-R\$ 45.000,00), "Custo da intervenção" (RCEI: R\$ 6.000,00-R\$ 24.000,00) e "Probabilidade de punção arterial acidental grave quando utilizada a técnica de ultrassonografia" (RCEl: R\$ 13.000,00$\mathrm{R} \$ 18.000,00)$.

Quanto à definição da disposição a pagar (WTP) da intervenção proposta, foi elaborada a curva de aceitabilidade (Figura 3B), identificando o valor em que o procedimento realizado sob auxílio do ultrassom se torna mais aceitável em relação à técnica-padrão (R\$18.125,00), utilizando posteriormente esse valor como limiar para a razão de custo-efetividade.

$\mathrm{Na}$ análise de sensibilidade probabilística (Figura 3A), utilizando o mesmo intervalo anterior (bilateral de 50\% do valor da média de cada variável), evidenciou-se que $63,6 \%$ das simulações apresentaram uma RCEl inferior a WTP estimada. Destaca-se que apenas 8,9\% das simulações se mostraram "dominadas" (intervenção menos efetiva e com custo superior), em relação à técnica-padrão.

\section{Discussão}

O estudo propôs avaliar a factibilidade econômica da inserção de cateter em veia jugular interna guiada por ultrassonografia. Sabe-se que avaliações econômicas possibilitam auxiliar a tomada de decisão, oferecendo suporte aos gestores, além de informações aos órgãos reguladores relacionados às alternativas disponíveis, visando à alocação dos recursos de forma estruturada (Santos, 2010). Em um cenário ideal, a elaboração de estudos econômicos, como aqui executado, pode contribuir para a sustentabilidade do sistema de saúde (Novaes \& Elias, 2013).
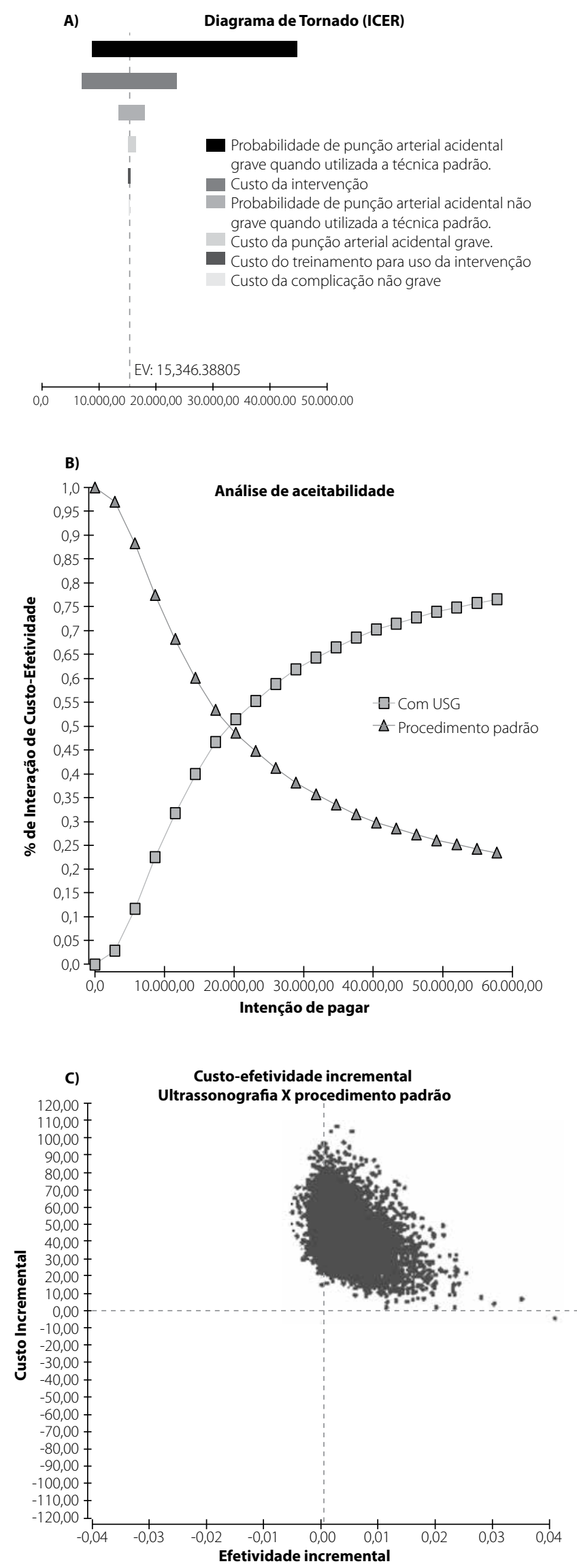

Figura 3. Análises de sensibilidade determinística (A); de aceitabilidade (B) e de sensibilidade probabilística (C). 
Nos últimos tempos, tem sido constatado aumento da utilização de diversas tecnologias médicas em procedimentos diferentes dos inicialmente preconizados pelos fabricantes. No caso do ultrassom, há todo um movimento de estímulo da sua empregabilidade não só para diagnósticos de imagem, mas também mediante o desenvolvimento de aparelhos cada vez mais acessíveis financeiramente e até mesmo portáteis, em cateterismos venosos, profundos e periféricos. Em razão desse uso não previamente considerado, as sociedades reguladoras têm se ajustado aos avanços tecnológicos, com enfoque na difusão da tecnologia. A título de exemplificação, a Sociedade Americana de Ecocardiografia e Anestesiologistas Cardiovasculares, desde o ano de 2012, tem apontado como "capacitado" o profissional que tiver realizado, sob supervisão, um montante de dez procedimentos. Essa regulamentação foi emitida no mesmo ano em que o Comitê Internacional de Acesso Vascular com Ultrassom publicou suas recomendações, também pretendendo incentivar o uso da tecnologia com esse fim (Gallotti, 2004).

Deve-se reiterar que a utilização do ultrassom para suportar o método de cateterismo venoso central está associada à elevação da probabilidade de sucesso da intervenção (Tsotsolis et al., 2015). A rápida execução do procedimento e a ausência de radiação ionizante também se configuram como vantagens da adoção da ultrassonografia (laredi \& Shigueoka, 2010). Por outro lado, a habilidade do executor, bem como o custo do equipamento, quando considerado um serviço desprovido dessa tecnologia, seriam possíveis desvantagens a serem reportadas. Esses detrimentos terão mínimo impacto no cenário do nosso estudo e baixo impacto quando extrapolado para situações reais, haja vista que a unidade estudada e diversas outras unidades de saúde normalmente já utilizam esse equipamento para fins clássicos (diagnósticos de imagem) e a capacitação dos profissionais na intervenção estudada não denotaria nem um longo período nem gastos exorbitantes, segundo pesquisa de mercado feita pelos autores. Ademais, como evidenciado pela análise de sensibilidade determinística, o custo do treinamento para os profissionais responsáveis não onerou significativamente, permitindo inferir que, apesar da necessidade de constante treinamento, ele não deve inviabilizar a implantação dessa tecnologia.

A presente pesquisa determinou o real investimento feito no procedimento para a inclusão da ultrassonografia, o qual onerou ( $\mathrm{R} \$ 53,81$ ) discretamente o modelo, quando comparado ao custo direto do procedimento associado à conduta terapêutica necessária nos casos de complicação. Tendo em vista esse aumento no custo, cita-se um estudo conduzido na Inglaterra (NICE, 2002) que atestou que, apesar da oneração da fonte pagadora em 10 libras (R\$ 48,20) por punção venosa guiada por ultrassom, os gastos oriundos das complicações foram reduzidos, em média, 2 libras $(R \$ 9,64)$ por procedimento.

Além desse quesito financeiro, esse estudo inglês (NICE, 2002) também reportou uma atenuação da razão de inci- dência de complicações advindas pelo cateterismo de veias centrais com o ultrassom na casa de 9\%. Espera-se que, com a utilização dessa tecnologia no Brasil, sobrevenha uma redução da taxa de 7,6\% descrita como proveniente de complicações em procedimentos hospitalares, principalmente, por saber-se que, desse montante, 66,7\% são classificadas como evitáveis (Mendes et al., 2009). Dessa maneira, a incorporação e a consequente utilização do ultrassom na rotina de realização de cateterismo venoso central pode proporcionar melhoria dos resultados atualmente existentes, viabilizando maior segurança para os pacientes e economia para os serviços, além da confiabilidade desse procedimento de tanta relevância para os prognósticos em saúde.

O estudo possui como limitação a não inclusão da compra e manutenção do equipamento, assim como dos custos administrativos da unidade. Tais limitantes não foram considerados na elaboração do modelo em virtude de o cenário analisado já possuir o equipamento em questão e sua consequentemente sua manutenção não ser considerada um gasto incremental. Outra limitação sucede em virtude da falta de informação brasileira específica a respeito da efetividade, sendo as utilizadas nesta pesquisa baseadas em estudos internacionais, podendo, portanto, diferir da efetividade no cenário brasileiro.

\section{Conclusão}

Diante de toda a exposição realizada, além da compreensão de que a intervenção seja custo-efetiva no cenário estudado, conclui-se que os achados aqui apresentados, em especial o valor da RCEl, proporcionarão segurança para a padronização de uso da ultrassonografia como orientadora do cateterismo venoso central de veia jugular interna. Acredita-se na necessidade da condução de futuros estudos brasileiros de efetividade sobre a referida técnica, aproximando cada vez mais as análises da realidade nacional.

\section{Referências bibliográficas}

AHRQ - Healthcare Research and Quality. Use of Real-Time Ultrasound Guidance During Central Line Insertio 2001.

Brasil. Compras Governamentais. Ministério do Planejamento, Orçamento e Gestão.

Brasil. Sistema de Gerenciamento da Tabela de Procedimentos, Medicamentos e Órteses/Próteses do Sistema Único de Saúde: Datasus.

Brass P, Hellmich M, Kolodziej L, Schick G, Smith AF. Ultrasound guidance versus anatomical landmarks for internal jugular vein catheterization. Cochrane Database Syst Rev. 2015;1:CD006962.

Gallotti RMD. Eventos adversos: o que são? Rev Assoc Méd Bras. 2004:50(2):114.

laredi W, Shigueoka DC. Exposição à radiação durante exames de imagem: dúvidas frequentes. Diagn Tratamento. 2010;15(3):143-5.

Kornbau C, Lee KC, Hughes GD, Firstenberg MS. Central line complications. Int J Crit IIIn Inj Sci. 2015;5(3):170-8.

Kusminsky RE. Complications of central venous catheterization. J Am Coll Surg. 2007;204(4):681-96. 
Mansfield PF, Hohn DC, Fornage BD, Gregurich MA, Ota DM. Complications and failures of subclavian-vein catheterization. N Engl J Med. 1994;331(26):1735-8.

Mendes W, Martins M, Rozenfeld S, Travassos C. The assessment of adverse events in hospitals in Brazil. Int J Qual Health Care. 2009;21(4):279-84.

Merrer J, De Jonghe B, Golliot F, Lefrant JY, Raffy B, Barre E, et al.; French Catheter Study Group in Intensive Care.. Complications of femoral and subclavian venous catheterization in critically ill patients: a randomized controlled trial. JAMA. 2001;286(6):700-7.

NICE - National Institute for Health and Care Excellence. Guidance on the use of ultrasound locating devices for placing central venous catheters. NICE technology appraisal guidance. 2002. Available from: https://www. nice.org.uk/guidance/ta49. Accessed on: May 20, 2019.
Novaes HMD, Elias FTS. Uso da avaliação de tecnologias em saúde em processos de análise para incorporação de tecnologias no Sistema Único de Saúde no Ministério da Saúde. Cad Saúde Pública. 2013;29(Suppl 1):s7-s16.

Petisco GM, Petisco ACGP, Fiato UAP, Santos FB. Cateterização Venosa Guiada por Ultrassom: Relato de Caso e Revisão da Literatura. Rev Bras Ecocardiogr Imagem Cardiovasc. 2013;26(3):228-35.

Santos VCC. As análises econômicas na incorporação de tecnologias em saúde: reflexões sobre a experiência brasileira [dissertação]. Rio de Janeiro: Escola Nacional de Saúde Pública Sergio Arouca; 2010.

Tsotsolis N, Tsirgogianni K, Kioumis I, Pitsiou G, Baka S, Papaiwannou A, et al. Pneumothorax as a complication of central venous catheter insertion. Ann Transl Med. 2015;3(3):40. 